\title{
A Comprehensive Addressing and Erudition on Blockchain in IOT Fortification
}

\author{
R. Priyadharsini M.C.A., M.Phil. \\ Department of Computer Applications, Sri Krishna Arts and Science, Coimbatore, TamilNadu, India
}

\begin{abstract}
Article Info

Volume 7, Issue 3

Page Number: 233-237

Publication Issue :

May-June-2021

\section{Article History}

Accepted : 15 May 2021

Published : 22 May 2021

Blockchain is the technology that provides security through its cryptography. IOT (Internet of Things) enhances the usage of software and hardware power in efficient way. The IOT Devices can be configured and controlled by blockchain. In this, the analysis of blockchain in IOT Security presented. The Key management is one of the biggest features for blockchain to be successful in the technology. As security is essential for any technology to be successful, the importance is considered and revealed about the security of IOT through blockchain technology. The features and considerations made would be useful for further research on blockchain in IOT Security.

Keywords : Blockchain, Internet of Things (IOT), Security, Key Mechanism.
\end{abstract}

\section{INTRODUCTION}

\subsection{Interpretation}

A blockchain is a digital record of transactions. The name comes from its structure, in which individual record, called block, are linked together in single list, called a chain. Blockchains are used for recording transaction made with crypto currencies, such as Bitcoin, and have many other applications [1].

\subsection{Uses of Blockchain}

The following [2] are the potential uses of Blockchain Technology.

- Payment Processing and money transfers.

- Monitor supply chains.

- Retail loyalty rewards programs.

- Digital IDs.

- Data sharing.
- Copyright and royalty protection.

- Digital voting.

- Real estate, land, and auto title transfers.

- Immutable data backup.

- Tax regulations and compliance.

- Worker's Rights.

- Medical recordkeeping.

- Weapons tracking.

- Wills or inheritance.

- Equality trading.

- Managing Internet of Things networks.

- Expenditure energy futures trading and compliance.

- Securing access to belongings

- Tracking Prescription drugs. 


\subsection{Blockchain Security}

The Blockchain technology secures data and network with the following features [3].

1. Distributed Data Database.

2. Encryption Technology.

3. Key Management.

\subsection{Internet of Things (IOT)}

According to Gartner [4], the Internet of Things is the network of physical objects that contain embedded technology to communicate and sense or interact with their states or external environment.

IOT is the technology that simplifies human life. In the IOT applications, many transactions of data over the network occur.

\subsection{IOT Security}

The IOT Security is accomplished in all aspects of its functionalities. Data and network should be secured. The following [5] are the basic components for the robust security of [IOT].

- Encryption

- Authentication

- Secure Storage

\subsection{Blockchain in IOT}

Blockchain in IOT is depicted in the following figure 1.

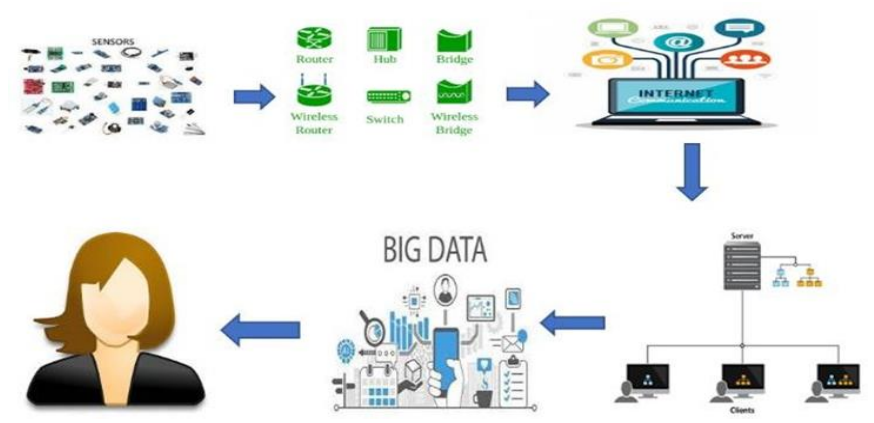

Figure 1. IOT with Blockchain Technology

The centralised network is replaced by decentralised network which would bring the following benefits.

- Secure authentication

- No tampering of data

- Distributed data share
The above introduction gives us about Block chain, Uses of Block chain, Block chain Security, Io T and its Security, Block chain in IOT. The rest of the paper is organised as follows. The following section 2 comprises the related work on Block chain on IOT security. Section 3 comprises Block chain on IOT Security. The conclusion is made in Section 4 with the suggestions regarding Block chain on IOT security for further research.

\section{LITERATURE REVIEW}

In [7], the author reveals about certain points on the block chain mechanism on IoT Security. The main advantage of using block chain is that it provides security to all layers and domain of IoT System.

The layer level security mechanisms are considered like device-level block chains, site-level block chains, gateway-level block chains, end-to-end block chains and analytical/storage- level block chains. IOT reference framework is framed for the clarity about security in layers.

The smart home system is considered for the block chain based in [8]. The BC (block chain)-Based Smart Home companies are transactions (Stores, Access, Monitor, Genesis (new), Remove), local block chain (tracks transactions and ensures policy), home miner (central process management includes transactions management includes transaction management, integration, transactions collected and appended to $\mathrm{BC})$, local storage.

The BC-Based Smart Home comprises the Initialization steps like Initialization (updating), transactions handling (Distributed key management, authorisation) and shared overlay (shared miner, Virtual Private Network (VPN)). Security analysis and Performance Evaluation are discussed under evaluation and analysis [8].

The symmetric encryption, hashing, transactions limitation, logging transactions and usage of policy header, shared keys are employed to satisfy 
requirements like confidentiality, Integrity, availability, user control and authorization respectively. The low overhead gives security and privacy.

The blockchain used for IOT has many key Ethereum nodes execute according to the benefits. It provides trust, reduce task, instructions. Innumerable platform available foe blockchain technology success. In [12], the author classifies application as follows in Table 1. TABLE 1: IOT -BLOCKCHAIN APPLICATIONS

\begin{tabular}{|c|c|c|}
\hline $\begin{array}{l}\text { APPLICA } \\
\text { TION }\end{array}$ & CLASSIFICATION & PLATFORM \\
\hline $\begin{array}{l}\text { LO3 } \\
\text { Energy }\end{array}$ & Energy microgrid & Ethereum \\
\hline ASEPT & $\begin{array}{lr}\text { Smart } & \text { contracts } \\
\text { involving } & \text { IOT } \\
\text { devices. } & \end{array}$ & Ethereum \\
\hline Slockit & $\begin{array}{l}\text { Renting/Selling/Shari } \\
\text { ng smart objects }\end{array}$ & Ethereum \\
\hline Aigang & $\begin{array}{l}\text { Insurance network } \\
\text { for lOT assets }\end{array}$ & Ethereum \\
\hline MyBit & Mybit & Ethereum \\
\hline $\begin{array}{l}\text { Aero } \\
\text { Token }\end{array}$ & 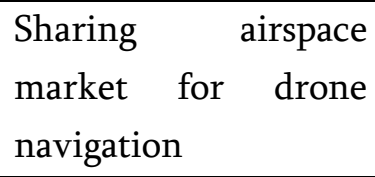 & Ethereum \\
\hline $\begin{array}{l}\text { Chain of } \\
\text { things }\end{array}$ & $\begin{array}{l}\text { Identity, Security and } \\
\text { Interoperability }\end{array}$ & Ethe \\
\hline Chronicled & $\begin{array}{ll}\text { Identity, } & \text { data } \\
\text { provenance } & \text { and } \\
\text { automation } & \end{array}$ & $\begin{array}{l}\text { Multiplatfor } \\
\text { m }\end{array}$ \\
\hline Modum & $\begin{array}{l}\text { Data integrity for the } \\
\text { supply chain }\end{array}$ & $\begin{array}{l}\text { Multiplatfor } \\
\mathrm{m}\end{array}$ \\
\hline $\begin{array}{l}\text { Riddle and } \\
\text { code }\end{array}$ & $\begin{array}{l}\text { Sharing and machine } \\
\text { economy }\end{array}$ & $\begin{array}{l}\text { Multiplatfor } \\
\mathrm{m}\end{array}$ \\
\hline $\begin{array}{l}\text { Blockchain } \\
\text { of things }\end{array}$ & $\begin{array}{l}\text { Secure connectivity } \\
\text { between IoT devices }\end{array}$ & $\begin{array}{l}\text { Multiplatfor } \\
\mathrm{m}\end{array}$ \\
\hline
\end{tabular}

The IOT devices can be configured and controlled by blockchain. Ethereum is used as blockchain platform [11]. Ethereum is massive shared computing system.
Because of its distributed capability, each device communicates with Ethereum. This provokes avoiding denial of services and malicious attacks.

Smart Contract is the concept used in blockchain. The programming languages used is solidity, serpent and LLL. Solidity is used as language and compiler. The codes are compiled and as byte codes deployed onto Ethereum.

There is an example considered by Pradip [9] for the detection of attacks in the IoT network in real time. DIST Block Net is the block chain technique to detect attacks in the IoT network in real time. The architecture of DIST Block satisfies the required principles with minimal overhead. Blockchain is integrated with IOT to reveal Perfect security in real time scenarios [10]. The IOT and blockchains is used to create secure shared economy distributed applications.

The blockchain used for IoT has many key benefits. It provides trust, reduce task, reduce costs and accelerate transactions [13]. Through all the above considered related work, the analysis is made and revealed the clear point about blockchain in IoT security. The following section 3 discusses about complete analysis on the blockchain in IoT security.

\section{BLOCK CHAIN IN IOT SECURITY}

Block chain can play a vital role in IOT security with its features. The following gives an overview about block chain in IoT security in various aspects.

The devices, processes and the humans using it access the diverse which contains logical supply chain, manufacture and health sector. An important key factor is that different types of sectors are attached and connected to these machines and devices. These devices have ability to sense and transmit the data and information from One node to another node (or) from one Cloud to another Cloud. Moreover, the data which is sensed by the devices are transmitted to the cloud server. Within a few units' time there may 
exists a complexity in the storing the date, processing. Here comes the emerging of the artificial intelligence techniques to extract the meaning information from the data which is sensed.

Block chain can potentially minimize the costs of the centralized systems. The internet of things can be used to build their distributed network. This would seek to identify many new applications in the wired and wireless applications. Here it is mandatory to seek and find out many new applications areas and vary high- quality reviews which describes the current state of the act. The following are

Block chain based IoT for the new network architecture.

Consensus algorithm design for wired /wireless environment.

Design for mobility limited resources and large - scale deployment.

\subsection{Architecture}

The architecture should the efficient usage of block chain in Io $\mathrm{T}$ security. Since block chain id decentralised, it provides benefits than traditional architecture [8], cloud and fog computing architectures, edge computing architecture, software defined networking [9] are few traditional architectures. These all have pitfalls like cyberattacks, lack of maintenance and software problems. Architecture is built to detect real-time attacks,increases through put and reduce delays.[6].

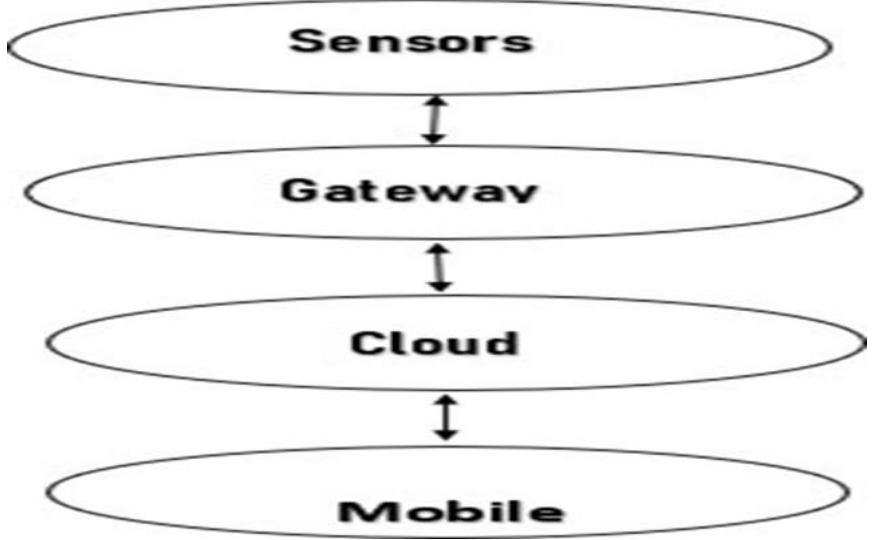

Figure 2. Blockchain Technology at data and network for secured flow

\subsection{Cryptographic Algorithms}

Security and privacy in a block chain are achieved by public-key cryptography [15].

A. Considerations:

- Computational Load

- Memory consumption

- Energy consumption

\section{B. Algorithms}

Algorithm works on key management. Public key is available in network for the flow of data and private key is for the accessibility of data. The smart contracts help in efficient protocol implementation. The protocol stack holds layers to provide accessibility, availability, accountability and efficient security.

Digital credentials are used for verification and validation for the communication over network. The block chain is a cryptographically linked list where the node has header, data and metadata. The ancillary metadata possess creator identity, signature and last block number. Algorithms provides secure in high priority for chain of blocks for transactions.

\section{IV.CONCLUSION}

The technologies provide ease of development of computer applications for human life. Blockchain and IOT plays vital role in the developments. blockchain technology is a great success with the emergence of bitcoin in 2008. Blockchain is an open, distributed ledger that can record transactions between two parties efficiently and in a verifiable and permanent way. The records on a block chain are secured through cryptography. IOT safeguards the activity of human life with machines without help of other human.

This paper provides an analysis through study of various related works on blockchain in IOT security. The various aspects like frameworks, key 
management, security algorithms for hardware and software, and programming languages is presented. This would lead a successful path for further research on blockchain in IOT security to frame an efficient application for human life.

\section{REFERENCES}

[1]. https://techterms.com/definition/blockchain

[2]. Scan Williams,"20Real-World Uses for Blockchain Technology”, TMF Ultra Long, 2018.

[3]. http://blockgeeks.com/guides/what-isblockchain- technology.

[4]. https://www.sas.com/en_in/insights/bigdata/internet-of- things.html

[5]. https://kyrio.com/wba-kyrio-partner-to-ensureopenroaming-security/

[6]. Nallapaneni Manoj Kumar, Pradeep Kumar Mallick, "Blockchain technology for security issues and challenges in IoT", Elsevier,2018.

[7]. Daniel Minoli, Bnedict Occhiogosso, "Blockchain mechanisms for Internet Security", ELSEVIER 2018.

[8]. Ali Dorri, Salil S. Kanhere, Raja Jurdak and Praveen Gauravaram, "Blockchain for IoT Security and privacy: The case study of a Smart Home", 2nd IEEE PERCOM Workshop ON Security Privacy and Trust in the Internet of Things 2017.

[9]. Pradip Kumar Sharma, Saurabh Singh, Young Sik Jeong and Jong Hyuk Park," DistBlockNet: A Distributed Blockchains -Based Secure SDN Architecture for IOT Networks." IEEE Communication Magazine, September 2017.

[10]. Steve Huckle, Ritupama Bhattacharya, Martin White and Natalia Beloff," Internet of Things, Blockchain and Shared Economy Applications", 1877-0509, Elsevier B.V,2016.

[11]. Seyoung Huh, Sangrae Cho, Soohyung Kim, "Managing IoT Devices using Blockchain Platform", ICACT2017.
[12]. Ana Reyna, Christian Martin, Jaime Chen, Enrique Soler, Manuel Diaz," On Blockchain and its integration with IOT. Challenges and opportunities, Elsevier B.V 2018.

[13]. https://www.i-scoop.eu/blockchain-distributedledger- technology/blockchain-iot/,"Blockchain opportunity and challenge".

[14]. Tiago M. Fernandz- Carames, Paula FragaLamas, "A review on the use of Blockchain in the Internet of Things", Volume 6, 2018, IEEE Access.

[15]. Olivier Alphand, Michele Amoretti, Timothy Claeys, Simone Dall 'Asta, Andrzej Duda, et al. IOT Chain: A Blockchain for the Internet of Things. IEEE Wireless Com- munications and networking Conference, Apr 2018, Barcelona, Spain,2018.

\section{Cite this Article}

R. Priyadharsini , "A Comprehensive Addressing and Erudition on Blockchain in IOT Fortification", International Journal of Scientific Research in Computer Science, Engineering and Information Technology (IJSRCSEIT), ISSN : 2456-3307, Volume 7 Issue 3, pp. 233-237, May-June 2021. Available at doi : https://doi.org/10.32628/CSEIT217344 Journal URL : https://ijsrcseit.com/CSEIT217344 